\title{
ON UNIFORMLY QUASICONFORMAL ANOSOV SYSTEMS
}

\author{
VICTORIA SADOVSKAYA
}

\begin{abstract}
We show that for any uniformly quasiconformal symplectic Anosov diffeomorphism of a compact manifold of dimension at least 4, its finite cover is $C^{\infty}$ conjugate to an Anosov automorphism of a torus. We also prove that any uniformly quasiconformal contact Anosov flow on a compact manifold of dimension at least 5 is essentially $C^{\infty}$ conjugate to the geodesic flow of a manifold of constant negative curvature.
\end{abstract}

\section{Introduction}

In this paper we study rigidity properties of uniformly quasiconformal Anosov systems. Our main goal is to obtain a complete classification of uniformly quasiconformal symplectic Anosov diffeomorphisms and contact Anosov flows up to a $C^{\infty}$ conjugacy. Such a classification for the diffeomorphisms is established in Theorems 1.1, and for flows in Theorem 1.2.

Theorem 1.1. Let $f$ be a $C^{\infty}$ symplectic Anosov diffeomorphism of a compact manifold $\mathcal{M}$, $\operatorname{dim} \mathcal{M} \geq 4$. If $f$ is uniformly quasiconformal on the unstable distribution, then its finite cover is $C^{\infty}$ conjugate to an Anosov automorphism of a torus.

Continuous time conformal Anosov systems were studied by M. Kanai in the special case of geodesic flows. He proved in [8] that the geodesic flow of a compact Riemannian manifold of negative curvature of dimension at least 3 is $C^{2}$ conjugate to the geodesic flow of a manifold of constant negative curvature under the assumption that either (a) the flow preserves a continuous conformal structure on the strong stable distribution, or (b) the flow satisfies $1 / 2$ pinching and preserves a bounded measurable conformal structure on the strong stable distribution. Later C. Yue showed in [15] that Kanai's result can be applied to uniformly quasiconformal geodesic flows of manifolds of negative curvature. He also noted that, by the main theorem of G. Besson, G. Courtois, and S. Gallot in [1], the existence of the above $C^{2}$ conjugacy implies that the manifold has constant negative curvature. Our next theorem generalizes Yue's result to the case of contact flows.

Theorem 1.2. Let $\varphi^{t}$ be a $C^{\infty}$ contact Anosov flow on a compact manifold $\mathcal{M}, \operatorname{dim} \mathcal{M} \geq 5$. If the flow is uniformly quasiconformal on the strong unstable

Received July 20, 2002. 
distribution, then it is essentially $C^{\infty}$ conjugate to the geodesic flow of a manifold of constant negative curvature.

More precisely, let $X$ be the vector field generating $\varphi^{t}$. Then there exists a closed 1-form $\alpha$ satisfying $1+\alpha(X)>0$ such that the flow generated by the vector field $Y=X /(1+\alpha(X))$ on a finite cover of $\mathcal{M}$ is $C^{\infty}$ conjugate to the geodesic flow of a compact manifold of constant negative curvature. The cohomology class of the form $\alpha$ is determined uniquely.

In a particular case when $\varphi^{t}$ is a geodesic flow of a compact Riemannian manifold $\mathcal{N}$ of negative curvature of dimension at least $3, \varphi^{t}$ is actually conjugate to the geodesic flow on a manifold of constant negative curvature. Then it follows from the main theorem of G. Besson, G. Courtois, and S. Gallot in [1] that $\mathcal{N}$ is a manifold of constant negative curvature. Thus we recover Yue's result for geodesic flows.

It was noted by Y. Benoist, P. Foulon, and F. Labourie in [2] that if $X$ generates a contact Anosov flow, then its time change generated by $Y$ as in Theorem 1.2 is also contact. Moreover, the inverse time change is of the same type, $X=Y /(1-\alpha(Y))$. So the flow $\varphi^{t}$ can be viewed as such a time change of a finite factor of the geodesic flow of a compact manifold of constant negative curvature.

We show in Section 3.4 that any smooth time change of a uniformly quasiconformal Anosov flow is also uniformly quasiconformal. Thus there exist smooth time changes of the geodesic flow of a compact manifold of constant negative curvature which are uniformly quasiconformal, but not $C^{\infty}$ conjugate to a time change of the above type. So the assumption that the flow is contact can not be omitted in Theorem 1.2.

Our approach to the proof of Theorems 1.1 and 1.2 is different from the approach developed by M. Kanai. We use results of Y. Benoist, P. Foulon, and F. Labourie, who proved in [2] and [3] that

(a) any symplectic Anosov diffeomorphism with $C^{\infty}$ Anosov splitting is $C^{\infty}$ conjugate to an automorphism of an infranilmanifold, and

(b) any contact Anosov flow with $C^{\infty}$ Anosov splitting is essentially $C^{\infty}$ conjugate (in the sense described in Theorem 1.2) to the geodesic flow of a locally symmetric manifold of negative curvature.

The main steps of our proof are Theorems 1.3 and 1.4 which may be of independent interest.

Theorem 1.3. Let $f$ be a topologically transitive $C^{\infty}$ Anosov diffeomorphism $\left(\varphi^{t}\right.$ be a topologically mixing $C^{\infty}$ Anosov flow) on a compact manifold $\mathcal{M}$ which is uniformly quasiconformal on the (strong) unstable distribution. Then it is conformal with respect to a Riemannian metric on this distribution which is Hölder continuous on $\mathcal{M}$ and $C^{\infty}$ along the leaves of the (strong) unstable foliation. 
The existence of such a metric for Anosov flows (and diffeomorphisms) was established by R. de la Llave in [10] under the assumption that the strong unstable subspaces $E^{s u}(x)$ can be continuously identified with $\mathbb{R}^{\operatorname{dim} E^{s u}}$ in such a way that the restriction of the differential to $E^{s u}$ is a scalar multiple of identity.

We use the above metric to establish smoothness of the (weak) stable distribution for uniformly quasiconformal systems.

Theorem 1.4. Let $f\left(\varphi^{t}\right)$ be a $C^{\infty}$ Anosov diffeomorphism (flow) on a compact manifold $\mathcal{M}$ with $\operatorname{dim} E^{u} \geq 2\left(\operatorname{dim} E^{s u} \geq 2\right)$. Suppose it is conformal with respect to a Riemannian metric on the (strong) unstable distribution which is continuous on $\mathcal{M}$ and $C^{\infty}$ along the leaves of the (strong) unstable foliation. Then the (weak) stable holonomy maps are conformal, and the (weak) stable distribution is $C^{\infty}$.

We note that in Theorems 1.3 and 1.4 we do not assume that the diffeomorphism is symplectic (the flow is contact).

We would like to thank Boris Kalinin and Ralf Spatzier for encouragement, support, and helpful discussions.

\section{Preliminaries}

In this section we briefly introduce the main notions used throughout this paper.

2.1. Anosov flows. Let $X$ be a smooth vector field on a compact Riemannian manifold $\mathcal{M}$, and let $\varphi^{t}: \mathcal{M} \rightarrow \mathcal{M}$ be the flow generated by $X$. The flow $\varphi^{t}$ is called Anosov if there exists a decomposition of the tangent bundle $T \mathcal{M}$ into three $\varphi^{t}$-invariant continuous subbundles $\mathbb{R} X, E^{s u}, E^{s s}$, and constants $C>0$, $\lambda>0$ such that for all $t \geq 0$,

$$
\begin{aligned}
& \left\|d \varphi^{-t}(v)\right\| \leq C e^{-\lambda t}\|v\| \text { for } v \in E^{s u}, \\
& \left\|d \varphi^{t}(w)\right\| \leq C e^{-\lambda t}\|w\| \text { for } w \in E^{s s} .
\end{aligned}
$$

The distributions $E^{s u}, E^{s s}, E^{u}=E^{s u} \oplus \mathbb{R} X$, and $E^{s}=E^{s s} \oplus \mathbb{R} X$ are called strong unstable, strong stable, weak unstable and weak stable. It is well-known that these distributions are tangential to the foliations $W^{s u}, W^{s s}, W^{u}$, and $W^{s}$ respectively (see, for example [9]). The leaves of these foliations are $C^{\infty}$ injectively immersed Euclidean spaces, but in general the distributions $E^{s u}$, $E^{s s}, E^{u}$, and $E^{s}$ are only Holder continuous transversally to the corresponding foliations.

Anosov diffeomorphisms are defined similarly (see [9]).

2.2. Contact flows. Let $\mathcal{M}$ be a smooth manifold of dimension $2 n+1$. A contact form $\theta$ on $\mathcal{M}$ is a smooth 1 -form such that $\theta \wedge(d \theta)^{n}$ is a volume form on $\mathcal{M}$. Let $\varphi^{t}$ be the flow on $\mathcal{M}$ generated by a smooth vector field $X$. The flow $\varphi^{t}$ is called contact if it preserves a contact form $\theta$, i.e. for any $t,\left(\varphi^{t}\right)^{*}(\theta)=\theta$, and $\theta(X)=1$. If the flow $\varphi^{t}$ is Anosov, then $\operatorname{ker} \theta=E^{s s} \oplus E^{s u}$. 
2.3. Symplectic diffeomorphisms. Let $\mathcal{M}$ be a smooth even-dimensional manifold. A symplectic form on $\mathcal{M}$ is a closed non-degenerate differential 2-form. A diffeomorphism $f: \mathcal{M} \rightarrow \mathcal{M}$ is called symplectic if it preserves a symplectic form $\omega$, i.e. $f^{*}(\omega)=\omega$.

2.4. Uniformly quasiconformal flows. Let $\varphi^{t}$ be an Anosov flow on a compact Riemannian manifold $\mathcal{M}$. We say that the flow is uniformly quasiconformal on the strong unstable distribution or uniformly $u$-quasiconformal if the quasiconformal distortion

$$
K(x, t)=\frac{\max \left\{\left\|d \varphi^{t}(v)\right\|: v \in E^{s u}(x),\|v\|=1\right\}}{\min \left\{\left\|d \varphi^{t}(v)\right\|: v \in E^{s u}(x),\|v\|=1\right\}}
$$

is uniformly bounded for all $t \in \mathbb{R}$ and $x \in \mathcal{M}$. This is equivalent to the classical definition of uniform quasiconformality, since

$$
K(x, t)=\limsup _{r \rightarrow 0} \frac{\sup \left\{d^{s u}\left(\varphi^{t} y, \varphi^{t} x\right): y \in S^{s u}(x, r)\right\}}{\inf \left\{d^{s u}\left(\varphi^{t} y, \varphi^{t} x\right): y \in S^{s u}(x, r)\right\}},
$$

where $d^{s u}$ is the induced metric along the $W^{s u}$ leaves and $S^{s u}(x, r)=\{y \in$ $\left.W^{s u}(x): d^{s u}(x, y)=r\right\}$.

If $K(x, t)=1$ for all $x$ and $t$, the flow is called $u$-conformal. The notions of $s$ conformality and uniform $s$-quasiconformality are defined similarly. If the flow is both uniformly $u$-quasiconformal ( $u$-conformal) and uniformly $s$-quasiconformal ( $s$-conformal) then it is called uniformly quasiconformal (conformal).

Note that if $\varphi^{t}$ is uniformly quasiconformal with respect to some Riemannian metric on $\mathcal{M}$, then it is uniformly quasiconformal with respect to any Riemannian metric on $\mathcal{M}$. Thus the notion does not depend on the choice of the metric.

2.5. Conformal structures. (See [8] for more details.) A conformal structure on $\mathbb{R}^{n}, n \geq 2$, is a class of proportional inner products. The space $C^{n}$ of conformal structures on $\mathbb{R}^{n}$ identifies with the space of real symmetric positive definite $n \times n$ matrices with determinant 1 which is isomorphic to $S L(n, \mathbb{R}) / S O(n, \mathbb{R})$. It is known that the space $C^{n}=S L(n, \mathbb{R}) / S O(n, \mathbb{R})$ carries a $G L(n, \mathbb{R})$-invariant metric for which $C^{n}$ is a Riemannian symmetric space of non-positive curvature. Any linear isomorphism of $\mathbb{R}^{n}$ induces an isometry of $C^{n}$.

Now, let $\varphi^{t}$ be an Anosov flow on a compact manifold $\mathcal{M}$. For each $x \in \mathcal{M}$, let $C^{s u}(x)$ be the space of conformal structures on $E^{s u}(x)$. Thus we obtain a bundle $C^{s u}$ over $\mathcal{M}$ whose fiber over $x$ is $C^{s u}(x)$. A continuous (smooth, measurable) section of $C^{s u}$ is called a continuous (smooth, measurable) conformal structure on $E^{s u}$. A measurable conformal structure $\tau$ is called bounded if the distance between $\tau(x)$ and $\tau_{0}(x)$ is uniformly bounded on $\mathcal{M}$, where $\tau_{0}$ is a continuous conformal structure on $\mathcal{M}$.

Similarly, we define a bundle $C^{u}$ and conformal structures for the case of an Anosov diffeomorphism of $\mathcal{M}$.

Clearly, a flow (diffeomorphism) is conformal with respect to a Riemannian metric if and only if it preserves the conformal structure associated with this metric. 


\section{Proofs of the Main Results}

3.1. Proof of Theorem 1.3. We prove the theorem for a uniformly $u$-quasiconformal Anosov flow. The proof and the intermediate statements can be easily modified for the case of diffeomorphism.

Let $\varphi^{t}$ be a uniformly $u$-quasiconformal Anosov flow on a compact manifold $\mathcal{M}$. Our goal is to obtain a metric on the strong unstable distribution with respect to which the flow is $u$-conformal. This metric will be Hölder continuous on $\mathcal{M}$ and $C^{\infty}$ along the leaves of the strong unstable foliation. To construct such a metric, we do not need to assume that the flow is contact.

We obtain the metric as follows. First we construct an invariant bounded measurable conformal structure, then a continuous invariant conformal structure. After that we use a statement on non-stationary linearization to show that this structure is actually $C^{\infty}$ along the leaves of the strong unstable foliation. Then we normalize the conformal structure using a smooth function to obtain a metric.

The next proposition follows from a theorem due to P. Tukia who established this result for an arbitrary quasiconformal group action in [14]. It was observed earlier by D. Sullivan in [13] for the case of discrete groups. Below we outline Tukia's proof for our case.

Proposition 3.1. Let $\varphi^{t}$ be a uniformly u-quasiconformal Anosov flow on a compact manifold $\mathcal{M}$. Then $\varphi^{t}$ preserves a bounded measurable conformal structure $\tilde{\tau}$ on the strong unstable distribution.

Proof. Let $\tau_{0}$ be a continuous conformal structure on $\mathcal{M}$. For $x \in \mathcal{M}$, consider the set $C(x)=\left\{\varphi^{-t}\left(\tau_{0}\left(\varphi^{t} x\right)\right), t \in \mathbb{R}\right\}$. Since the flow is uniformly $u$ quasiconformal, it is a bounded subset of $C^{s u}(x)$, the space of conformal structures on $E^{s u}(x)$. Since $C^{s u}(x)$ has non-positive curvature, there exists a uniquely determined ball of the smallest radius containing $C(x)$. We denote its center by $\tilde{\tau}(x)$. One can show that the conformal structure $\tilde{\tau}$ is invariant, bounded and measurable.

The following statement was obtained jointly with Boris Kalinin.

Proposition 3.2. Let $\varphi^{t}$ be a topologically mixing Anosov flow on a compact manifold $\mathcal{M}$ which preserves a bounded measurable conformal structure $\tilde{\tau}$ on the strong unstable distribution. Then there exists a Hölder continuous $\varphi^{t}$-invariant conformal structure $\tau$ on the strong unstable distribution, which coincides with $\tilde{\tau}$ on a set of full (Bowen-Margulis) measure.

Remark 3.1. Since a topologically transitive Anosov diffeomorphism of a compact connected manifold is topologically mixing, it is enough to assume topological transitivity for the discrete-time analog of this proposition.

Proof. To simplify the notations, we identify tangent spaces at close points. This allows us to conveniently compare conformal structures at different points. 
Since the distribution $E^{s u}$ is only Hölder continuous (with some Hölder exponent $\alpha>0$ ), the identification is also Hölder continuous, and hence $d f$ is Hölder continuous with respect to the identification. The map $d\left(\varphi^{-1}\right)_{x}$ induces an isometry $T_{x}$ from $C^{s u}(x)$, the space of conformal structures on $E^{s u}(x)$, to $C^{s u}\left(\varphi^{-1} x\right)$, the space of conformal structures on $E^{s u}\left(\varphi^{-1} x\right)$. For $x \in \mathcal{M}$, we denote by $\tilde{\tau}(x)$ the conformal structure on $E^{s u}(x)$.

First we estimate the distance between the conformal structures at $x \in \mathcal{M}$ and at a nearby point $y \in W^{s s}(x)$. Let $x_{i}=\varphi^{i}(x)$, and $y_{i}=\varphi^{i}(y)$. Since the conformal structure $\tilde{\tau}$ is invariant, $\tilde{\tau}(x)=T_{x_{1}}\left(\tilde{\tau}\left(x_{1}\right)\right)$ and $\tilde{\tau}(y)=T_{y_{1}}\left(\tilde{\tau}\left(y_{1}\right)\right)$. Since $T$ is an isometry, we obtain

$$
\begin{aligned}
\operatorname{dist}(\tilde{\tau}(x), \tilde{\tau}(y)) & =\operatorname{dist}\left(T_{x_{1}}\left(\tilde{\tau}\left(x_{1}\right), T_{y_{1}}\left(\tilde{\tau}\left(y_{1}\right)\right)\right)\right. \\
& \leq \operatorname{dist}\left(T_{y_{1}}\left(\tilde{\tau}\left(x_{1}\right), T_{y_{1}}\left(\tilde{\tau}\left(y_{1}\right)\right)\right)+\operatorname{dist}\left(T_{x_{1}}\left(\tilde{\tau}\left(x_{1}\right), T_{y_{1}}\left(\tilde{\tau}\left(x_{1}\right)\right)\right)\right.\right. \\
& \left.=\operatorname{dist}\left(\tilde{\tau}\left(x_{1}\right), \tilde{\tau}\left(y_{1}\right)\right)\right)+\operatorname{dist}\left(\tilde{\tau}\left(x_{1}\right), T_{x_{1}}^{-1} T_{y_{1}}\left(\tilde{\tau}\left(x_{1}\right)\right)\right) .
\end{aligned}
$$

Since $T$ depends Hölder continuously on the base point, and $\tilde{\tau}$ is bounded,

$$
\left.\operatorname{dist}(\tilde{\tau}(x), \tilde{\tau}(y)) \leq \operatorname{dist}\left(\tilde{\tau}\left(x_{1}\right), \tilde{\tau}\left(y_{1}\right)\right)\right)+K_{1} \cdot \operatorname{dist}\left(x_{1}, y_{1}\right)^{\alpha} .
$$

As the leaves of the strong stable foliation are contracted exponentially, we obtain by induction that

$$
\begin{aligned}
\operatorname{dist}(\tilde{\tau}(x), \tilde{\tau}(y)) & \leq \operatorname{dist}\left(\tilde{\tau}\left(x_{n}\right), \tilde{\tau}\left(y_{n}\right)\right)+\sum_{i=1}^{n} K_{1} \cdot \operatorname{dist}\left(x_{i}, y_{i}\right)^{\alpha} \\
& \leq \operatorname{dist}\left(\tilde{\tau}\left(x_{n}\right), \tilde{\tau}\left(y_{n}\right)\right)+K_{1} K_{2} \cdot \operatorname{dist}(x, y)^{\alpha}
\end{aligned}
$$

where $K_{1} K_{2}=K^{s s}$ is a constant independent of $x, y$, and $n$.

Since the flow $\varphi^{t}$ is topologically mixing, there exists the Bowen-Margulis measure $\mu$ on $\mathcal{M}$. This measure has full support, and is mixing with respect to the flow and its time-one map.

Since the conformal structure $\tilde{\tau}$ is measurable, it is uniformly continuous on a set $A \subset \mathcal{M}$ with $\mu(A)>1 / 2$. It follows from the Birkhoff ergodic theorem that the set of points for which the frequency of visiting $A$ equals $\mu(A)$ has full measure. We denote this set by $G$. If both $x$ and $y$ are in $G$, then there exists a sequence $\left(n_{k}\right)$ such that $x_{n_{k}} \in A$ and $y_{n_{k}} \in A$. If in addition $x$ and $y$ lie on the same strong stable leaf, then $\operatorname{dist}\left(x_{n_{k}}, y_{n_{k}}\right) \rightarrow 0$, and hence $\operatorname{dist}\left(\tilde{\tau}\left(x_{n_{k}}\right), \tilde{\tau}\left(y_{n_{k}}\right)\right) \rightarrow 0$. Thus, we obtain

$$
\operatorname{dist}(\tilde{\tau}(x), \tilde{\tau}(y)) \leq K^{s s} \cdot \operatorname{dist}(x, y)^{\alpha} .
$$

By a similar argument, $\operatorname{dist}(\tilde{\tau}(x), \tilde{\tau}(z)) \leq K^{s u} \cdot \operatorname{dist}(x, z)^{\alpha}$ for any two nearby points $x, z \in G$ lying on the same strong unstable leaf. Since $\tilde{\tau}$ is invariant under the flow, it follows that $\operatorname{dist}(\tilde{\tau}(x), \tilde{\tau}(z)) \leq K^{u} \cdot \operatorname{dist}(x, z)^{\alpha}$ for any two nearby points $x, z \in G$ on the same weak unstable leaf.

Consider a small open set in $\mathcal{M}$ with a product structure. For almost all local strong stable leaves, the set of points of $G$ on the leaf has full conditional measure. Consider points $x, y \in G$ lying on two such strong stable leaves. Let $H_{x, y}$ be the weak unstable holonomy map between $W^{s s}(x)$ and $W^{s s}(y)$. Since 
the Bowen-Margulis measure is invariant under holonomy maps, there exists a point $z \in W^{s s}(x) \cap G$ close to $x$ such that $H_{x, y}(z)$ is also in $G$. By the above argument,

$$
\begin{aligned}
\operatorname{dist}(\tilde{\tau}(x), \tilde{\tau}(z)) & \leq K^{s s} \cdot \operatorname{dist}(x, z)^{\alpha}, \\
\operatorname{dist}\left(\tilde{\tau}(z), \tilde{\tau}\left(H_{x, y}(z)\right)\right) & \leq K^{u} \cdot \operatorname{dist}\left(z, H_{x, y}(z)\right)^{\alpha}, \quad \text { and } \\
\operatorname{dist}\left(\tau\left(H_{x, y}(z)\right), \tau(y)\right) & \leq K^{s s} \cdot \operatorname{dist}\left(H_{x, y}(z), y\right)^{\alpha} .
\end{aligned}
$$

Since the points $x, y$, and $z$ are close, it is clear from the local product structure that

$$
\operatorname{dist}(x, z)^{\alpha}+\operatorname{dist}\left(z, H_{x, y}(z)\right)^{\alpha}+\operatorname{dist}\left(H_{x, y}(z), y\right)^{\alpha} \leq \tilde{K} \cdot \operatorname{dist}(x, y)^{\alpha} .
$$

Hence, we obtain $\operatorname{dist}(\tilde{\tau}(x), \tilde{\tau}(y)) \leq K \tilde{K} \cdot \operatorname{dist}(x, y)^{\alpha}$.

So $\tilde{\tau}$ is Hölder continuous on a set $B$ of full measure. We can assume that $B$ is invariant by considering $\bigcap_{n=-\infty}^{\infty} f^{n}(B)$. Since $B$ is dense, we can extend $\tilde{\tau}$ from $B$ and obtain an invariant Hölder continuous conformal structure $\tau$ on $\mathcal{M}$.

Remark 3.2. The proof works if we start with a measurable conformal structure which is essentially bounded with respect to the Bowen-Margulis measure or the absolutely continuous invariant measure.

To show that the conformal structure $\tau$ constructed in Proposition 3.2 is smooth along the strong unstable leaves we use the following corollary of Proposition 4.1 (see Section 4).

Proposition 3.3. Let $f$ be a uniformly u-quasiconformal Anosov diffeomorphism of a compact manifold $\mathcal{M}$. Then for any $x \in \mathcal{M}$ there exists a $C^{\infty}$ diffeomorphism $h_{x}: W^{u}(x) \rightarrow E^{u}(x)$ such that

(i) $h_{f x} \circ f=d f_{x} \circ h_{x}$,

(ii) $h_{x}(x)=0$ and $\left(d h_{x}\right)_{x}$ is the identity map,

(iii) $h_{x}$ depends continuously on $x$ in $C^{\infty}$ topology.

Let $\varphi^{t}$ be a uniformly u-quasiconformal Anosov flow on a compact manifold $\mathcal{M}$. Then for any $x \in \mathcal{M}$ there exists a $C^{\infty}$ diffeomorphism $h_{x}: W^{s u}(x) \rightarrow E^{s u}(x)$ satisfying

(i') $h_{\varphi^{1} x} \circ \varphi^{1}=d \varphi_{x}^{1} \circ h_{x}$, and (ii), (iii) as above.

Recall that $\tau$ is a continuous invariant conformal structure on the strong unstable distribution. Now we show that $\tau$ is actually $C^{\infty}$ along the leaves. For each $x \in \mathcal{M}$ we extend the conformal structure $\tau(x)$ at $0 \in E^{s u}(x)$ to all other points of $E^{s u}(x)$ via translations. We denote this constant (translationinvariant) conformal structure on $E^{s u}(x)$ by $\sigma$. Since the conformal structure $\tau$ is $\varphi^{t}$-invariant, $\sigma$ is $d \varphi^{t}$-invariant.

For any map $g$ and conformal structure $\rho(x)$ at a point $x \in \mathcal{M}$, we denote by $g(\rho(x))$ the push forward of $\rho(x)$ to the point $g(x)$. 
Lemma 3.1. The map $h_{x}$ takes $\tau$ on $W_{x}^{\text {su }}$ into $\sigma$ on $E^{s u}(x)$, and hence $\tau$ is $C^{\infty}$ along the strong unstable leaves.

Proof. We need to show that for any $y \in W^{s u}(x), h_{x}(\tau(y))=\sigma\left(h_{x}(y)\right)$. To do this we flow back in time. First we note that for any $\epsilon>0$ there exists $n>0$ such that

$$
\operatorname{dist}\left(h_{\varphi^{-n} x}\left(\tau\left(\varphi^{-n} y\right)\right), \sigma\left(h_{\varphi^{-n} x}\left(\varphi^{-n}(y)\right)\right)<\epsilon .\right.
$$

Indeed, it follows from Proposition 3.3 (iii) that the restrictions of the derivative of $h_{x}$ to the ball of radius 1 around $x$ in $W^{s u}(x), x \in \mathcal{M}$, form an equicontinuous family. Hence if $\varphi^{-n} y$ is sufficiently close to $\varphi^{-n} x$, then $d h_{\varphi^{-n} x}\left(\varphi^{-n} y\right)$ is close to $d h_{\varphi^{-n} x}\left(\varphi^{-n} x\right)$, which is identity. Thus, $h_{\varphi^{-n} x}\left(\tau\left(\varphi^{-n} y\right)\right)$ is close to $h_{\varphi^{-n} x}\left(\tau\left(\varphi^{-n} x\right)\right)$, and by definition of $\sigma$,

$$
h_{\varphi^{-n} x}\left(\tau\left(\varphi^{-n} x\right)\right)=\sigma\left(h_{\varphi^{-n} x}\left(\varphi^{-n} x\right)\right)=\sigma\left(h_{\varphi^{-n} x}\left(\varphi^{-n} y\right)\right) .
$$

To obtain the following equalities, we note that $d \varphi^{n}$ induces an isometry between the spaces of conformal structures, $\tau$ is $\varphi$-invariant, $\sigma$ is $d \varphi$-invariant, and $h_{x}(y)=d \varphi^{n}\left(h_{\varphi^{-n} x}\left(\varphi^{-n}(y)\right)\right.$ by Proposition $3.3\left(\mathrm{i}^{\prime}\right)$. Thus,

$$
\begin{aligned}
\epsilon & >\operatorname{dist}\left(h_{\varphi^{-n} x}\left(\tau\left(\varphi^{-n} y\right)\right), \sigma\left(h_{\varphi^{-n} x}\left(\varphi^{-n}(y)\right)\right)\right. \\
& =\operatorname{dist}\left(d \varphi^{n}\left(h_{\varphi^{-n} x}\left(\tau\left(\varphi^{-n} y\right)\right)\right), d \varphi^{n}\left(\sigma\left(h_{\varphi^{-n} x}\left(\varphi^{-n}(y)\right)\right)\right)\right. \\
& =\operatorname{dist}\left(d \varphi ^ { n } \left(h_{\varphi^{-n} x}\left(\varphi^{-n}(\tau(y))\right), \sigma\left(d \varphi^{n}\left(h_{\varphi^{-n} x}\left(\varphi^{-n}(y)\right)\right)\right)\right.\right. \\
& =\operatorname{dist}\left(h_{x}(\tau(y)), \sigma\left(h_{x}(y)\right)\right) .
\end{aligned}
$$

As the above holds for any $\epsilon>0$, it follows that $h_{x}(\tau(y))=\sigma\left(h_{x}(y)\right)$.

Since the conformal structure $\sigma$ is constant on $E^{s u}(x)$ and $h_{x}$ is a $C^{\infty}$ diffeomorphism, we conclude that $\tau$ is $C^{\infty}$ along the strong unstable leaves.

Thus, we obtained an invariant conformal structure on the strong unstable distribution which is continuous on $\mathcal{M}$ and $C^{\infty}$ along the strong unstable leaves. Since a conformal structure is a class of proportional inner products, we normalize it using a $C^{\infty}$ function on $\mathcal{M}$ to obtain a Riemannian metric. So we have constructed a Riemannian metric on the strong unstable distribution with respect to which the flow is $u$-conformal. This metric is Hölder continuous on $\mathcal{M}$ and $C^{\infty}$ along the strong unstable leaves.

3.2. Proof of Theorem 1.4. First we show that for a uniformly $u$-quasiconformal Anosov system the (weak) unstable distribution is $C^{1}$. This result seems to be well-accepted, however, due to the lack of precise reference, we indicate how it can be obtained using an argument in [6].

Proposition 3.4. Let $f\left(\varphi^{t}\right)$ be a uniformly u-quasiconformal Anosov diffeomorphism (flow) on a compact manifold $\mathcal{M}$. Then the (weak) stable distribution is $C^{1}$.

Proof. In Section 4 of [6] M. Hirsch and C. Pugh proved, using the $C^{r}$ section theorem, that the strong stable distribution is $C^{1}$ for the geodesic flow on a negatively curved surface. As easily follows from the remark at the end of 
their proof, the argument works in higher dimension for a contact $u$-conformal Anosov flow. Now, using Theorem 1.3 we conclude that the same result holds for a uniformly $u$-quasiconformal contact Anosov flow (this can also be seen more directly by considering time- $t$ map for sufficiently large $t$ ). Clearly, the argument works for the case of a uniformly $u$-quasiconformal Anosov diffeomorphism. For the case of flow a slight modification of the argument is needed since we don't assume that the flow is contact, and while $E^{u}$ is $C^{1}, E^{s u}$ is not necessarily smooth.

Consider the bundle $E=E^{s u} \oplus E^{s s}$. Since $E$ is Hölder continuous, we can approximate it by a smooth bundle $\tilde{E}$. Let $\hat{E}^{s u}=\tilde{E} \cap\left(E^{s u} \oplus \mathbb{R} X\right), \quad \hat{E}^{s s}=$ $\tilde{E} \cap\left(E^{s s} \oplus \mathbb{R} X\right)$, and let $\tilde{E}^{s u}$ and $\tilde{E}^{s s}$ be smooth subbundles of $\tilde{E}$ approximating $\hat{E}^{s u}$ and $\hat{E}^{s s}$ respectively.

Since $\tilde{E}$ is not invariant, instead of $d \varphi^{1}$ we consider the map $T: \tilde{E} \rightarrow \tilde{E}$, $T_{x}=\pi_{\varphi^{1} x} \circ d \varphi_{x}^{1}$, where $\pi_{\varphi^{1} x}$ is the projection of the tangent space at $\varphi^{1} x$ onto $\tilde{E}_{\varphi^{1} x}$ along the flow direction. As in section 4 of [6], one can see that $\hat{E}^{s u}$ is of class $C^{1}$. Hence the weak stable distribution $E^{u}=E^{s u} \oplus \mathbb{R} X=\hat{E}^{s u} \oplus \mathbb{R} X$ is also of class $C^{1}$.

We prove Theorem 1.4 for the case of flow. We denote by $\tau$ the conformal structure associated with the metric given in the theorem.

Let $x, y$ be two nearby points in $\mathcal{M}$. Consider the local holonomy map $H_{x, y}$ : $W^{s u}(x) \rightarrow W^{s u}(y)$ along the weak stable foliation: $z \in W^{s u}(x) \mapsto H_{x, y}(z)=$ $W^{s u}(y) \cap W^{s}(z)$.

First we show that the local holonomy maps $H_{x, y}$ are conformal. Since the weak stable distribution is $C^{1}$, the holonomy maps are uniformly $C^{1}([12])$. We will show that $H_{x, y}$ preserves the conformal structure $\tau$, i.e. $H_{x, y}(\tau(z))=\tau\left(z_{1}\right)$, where $z_{1}=H_{x, y}(z)$. Let $z_{2}=\varphi^{t_{0}}(z)$ be the point on $W^{s s}\left(z_{1}\right)$ with the smallest $\left|t_{0}\right|$. Then $H_{x, y}=H_{z_{2}, z_{1}} \circ H_{z, z_{2}}$, moreover, $H_{z, z_{2}}=\varphi^{t_{0}}$. Since $\varphi^{t}$ preserves the conformal structure, it suffices to show that $H_{z_{2}, z_{1}}\left(\tau\left(z_{2}\right)\right)=\tau\left(z_{1}\right)$. Note that $H_{z_{2}, z_{1}}=\varphi^{-t} \circ H_{\varphi^{t} z_{2}, \varphi^{t} z_{1}} \circ \varphi^{t}$. Since $z_{2} \in W^{s s}\left(z_{1}\right), \varphi^{t} z_{1}$ is close to $\varphi^{t} z_{2}$ for large $t$, and hence $d H_{\varphi^{t} z_{2}, \varphi^{t} z_{1}}$ is close to identity. Since $\tau$ is continuous, $\tau\left(\varphi^{t} z_{2}\right)$ is close to $\tau\left(\varphi^{t} z_{1}\right)$. Thus, $H_{\varphi^{t} z_{2}, \varphi^{t} z_{1}}\left(\tau\left(\varphi^{t} z_{2}\right)\right)$ is close to $\tau\left(\varphi^{t} z_{1}\right)$. Since $\varphi^{-t}$ induces an isometry between the spaces of conformal structures on $E^{s u}\left(\varphi^{t} z_{1}\right)$ and on $E^{s u}\left(z_{1}\right)$, we conclude that $H_{z_{2}, z_{1}}\left(\tau\left(z_{2}\right)\right)$ is close to $\tau\left(z_{1}\right)$. By letting $t \rightarrow \infty$, we can see that $H_{z_{2}, z_{1}}$ takes $\tau\left(z_{2}\right)$ into $\tau\left(z_{1}\right)$. Thus we conclude that the map $H_{x, y}$ is conformal.

Next we show that the maps $H_{x, y}$ are uniformly $C^{\infty}$, i.e. they are $C^{\infty}$ and their derivatives depend continuously on $y$. Fix $x \in \mathcal{M}$, and let $y$ be a point in a neighborhood $U$ of $x$. Let $g_{y}: E^{s u}(y) \rightarrow \mathbb{R}^{n}$ be a linear map which takes the metric on $E^{s u}(y)$ into the standard metric on $\mathbb{R}^{n}$. Clearly, $g_{y}$ can be chosen in such a way that it depends continuously on $y$ in $U$. Consider the map

$$
G_{x, y}=g_{y} \circ h_{y} \circ H_{x, y} \circ h_{x}^{-1} \circ g_{x}^{-1}: V \rightarrow \mathbb{R}^{n},
$$


where $V$ is an open set in $\mathbb{R}^{n}$ containing 0 , and $h_{x}$ is the map defined in Proposition 3.3. Since $G_{x, y}: V \rightarrow \mathbb{R}^{n}$ is a conformal $C^{1}$ diffeomorphism, it is $C^{\infty}$. Indeed, if $n>2$, it is a composition of a conformal affine map and an inversion, and if $n=2$ it is complex analytic. Then it also follows that that as the maps $G_{x, y}$ depend continuously on $y$, they depend continuously on $y$ in $C^{\infty}$ topology. Since $g_{y}$ are linear maps that depend continuously on $y$, they also depend continuously on $y$ in $C^{\infty}$ topology, and so does $h_{y}$ by Proposition 3.3. Hence, $H_{x, y}$ depends continuously on $y$ in $C^{\infty}$ topology.

Lemma 3.2. If the weak stable holonomies are uniformly $C^{\infty}$ (in the sense described above), then the weak stable distribution is $C^{\infty}$.

This statement is similar to Theorem 6.1 (ii) in [12], however in the theorem the holonomies are considered between the leaves of some smooth transverse foliation, while in our case the strong unstable foliation is not necessarily smooth.

Proof. As above, we fix a point $x \in \mathcal{M}$ and consider a neighborhood $U$ of $x$. We choose a $C^{\infty}$ coordinate chart $\Phi: U \rightarrow \mathbb{R}^{m} \times \mathbb{R}^{n}$ such that $\Phi(x)=0$, $\Phi\left(W^{s}(x) \cap U\right) \subset \mathbb{R}^{m}$, and $\Phi\left(W^{s u}(x) \cap U\right) \subset \mathbb{R}^{n}$. Let $p=(y, z) \in \mathbb{R}^{m} \times \mathbb{R}^{n}$. Consider the map

$$
\Psi(p)=\left(y, W^{s}(p) \cap W^{s u}(0)\right)=\left(y, H_{p, 0}(p)\right) .
$$

This map straightens the leaves of the weak stable foliation. Since the leaves of the weak stable foliation are uniformly $C^{\infty}$, the function $\Psi$ is uniformly $C^{\infty}$ on these leaves. Since the weak stable holonomy maps are uniformly $C^{\infty}$, as well as the leaves of the strong unstable foliation, $\Psi$ is uniformly $C^{\infty}$ on the leaves of the strong unstable foliation. Now it follows from the theorem established by Journé in [7] that $\Psi$ is $C^{\infty}$. This implies that the weak stable foliation (and the weak stable distribution) is $C^{\infty}$.

This completes the proof of Theorem 1.4.

3.3. Proof of Theorems 1.1 and 1.2. Let $f\left(\varphi^{t}\right)$ be a uniformly $u$-quasiconformal Anosov diffeomorphism (flow). Above we established that it is $u$ conformal with respect to some Riemannian metric on the (strong) unstable distribution, and that the (weak) stable distribution is $C^{\infty}$.

Now we assume that the flow is contact (the diffeomorphism is symplectic). The next statement shows that in this case the diffeomorphism (flow) is also $s$ conformal, i.e. it is conformal. This was observed by M. Kanai in [8] for contact flows. We include the argument for the sake of completeness.

Lemma 3.3. If a symplectic Anosov diffeomorphism (a contact Anosov flow) is $u$-conformal with respect to some continuous Riemannian metric on $E^{u}\left(E^{s u}\right)$, then it is s-conformal with respect to some continuous Riemannian metric on $E^{s}\left(E^{s s}\right)$. 
Proof. We present the proof for the case of flow. Similar argument works for the case of diffeomorphism.

The Riemannian metric on the unstable bundle $E^{s u}$ induces the dual metric on its dual bundle $\left(E^{s u}\right)^{*}$. The conformal structure on $\left(E^{s u}\right)^{*}$ associated with this metric is invariant under $\left(d \varphi^{t}\right)^{*}$.

Let $\theta$ be the contact form on $\mathcal{M}$. Then the restriction of its exterior derivative $d \theta$ to the subbundle $E=E^{s u} \oplus E^{s s}$ is a symplectic form on $E$. The splitting of $E$ into $E^{s u}$ and $E^{s s}$ is Lagrangian for $d \theta$, that is $d \theta\left(v_{1}, v_{2}\right)=0$ for any $v_{1}, v_{2} \in E^{s u}$, $d \theta\left(w_{1}, w_{2}\right)=0$ for any $w_{1}, w_{2} \in E^{s s}$, and $\operatorname{dim} E^{s u}=\operatorname{dim} E^{s s}$. Therefore, the natural map from $E^{s s}$ to $\left(E^{s u}\right)^{*}: w \mapsto f(v)=d \theta(v, w)$ is an isomorphism. Thus, the above metric on $\left(E^{s u}\right)^{*}$ induces a continuous metric on $E^{s s}$. Since $\theta$ is invariant, the associated conformal structure on $E^{s s}$ is also invariant. Thus, the flow is $s$-conformal.

It is known that symplectic Anosov diffeomorphisms and contact Anosov flows are topologically mixing. Now it is clear that Theorems 1.3 and 1.4 imply that for a uniformly $u$-quasiconformal symplectic Anosov diffeomorphism $f$ (contact Anosov flow $\varphi^{t}$ ) both (weak) stable and (weak) unstable distributions are $C^{\infty}$.

Note that for a contact flow the distribution $E^{s u}\left(E^{s s}\right)$ is smooth if and only if $E^{u}\left(E^{s}\right)$ is smooth, since $E^{s u}=E^{u} \cap \operatorname{ker} \theta\left(E^{s s}=E^{s} \cap \operatorname{ker} \theta\right)$, where $\theta$ is the contact form.

For the case of diffeomorphism, the result in [3] implies that $f$ is $C^{\infty}$-conjugate to an Anosov automorphism of an infranilmanifold. Since the diffeomorphism is uniformly quasiconformal, the corresponding nilpotent group has to be abelian. Indeed, if the group is not abelian, then the Anosov automorphism must have at least two unstable Lyapunov exponents and thus can not be uniformly quasiconformal. Since the group is abelian the infranilmanifold is finitely covered by a torus.

For the case of flow, it follows from Theorem 1 in [2] that that $\varphi^{t}$ is essentially $C^{\infty}$ conjugate (in the sense described in Theorem 1.2) to the geodesic flow of a locally symmetric manifold of strictly negative curvature. Proposition 3.5 below implies that the geodesic flow on this locally symmetric manifold has to be uniformly quasiconformal, and hence the manifold has constant negative curvature. Indeed, the geodesic flow of a locally symmetric manifold of non-constant strictly negative curvature has more than one unstable Lyapunov exponent, and thus it can not be uniformly quasiconformal.

This completes the proof of Theorems 1.1 and 1.2.

3.4. Smooth time changes. In Theorem 1.2 we assumed that the flow is contact. The next proposition shows that this assumption can not be omitted since any smooth time change of a uniformly $u$-quasiconformal Anosov flow is also uniformly $u$-quasiconformal. Note that any smooth time change of an Anosov flow is also Anosov. 
Proposition 3.5. Let $\varphi^{t}$ be a uniformly u-quasiconformal Anosov flow on a compact manifold $\mathcal{M}$, and let $\tilde{\varphi}^{t}$ be its smooth time change. Then $\tilde{\varphi}^{t}$ is also uniformly u-quasiconformal.

Proof. Fix a Riemannian metric on $\mathcal{M}$. Note that the orbits of $\varphi^{t}$ and of $\tilde{\varphi}^{t}$ are the same, and their weak unstable distributions $E^{u}$ and $\tilde{E}^{u}$ are also the same. Denote by $E^{s u}$ and $\tilde{E}^{s u}$ the strong unstable distributions of $\varphi^{t}$ and $\tilde{\varphi}^{t}$ respectively. Since $\mathcal{M}$ is compact, and both $E^{s u}$ and $\tilde{E}^{s u}$ are transversal to the flow direction in $E^{u}=\tilde{E}^{u}$, the angle they form with each other is bounded above by some $\alpha<\pi / 2$.

We will show that the quasiconformal distortion of $\tilde{\varphi}^{t}, \tilde{K}(t, x)$, is uniformly bounded for all $x \in \mathcal{M}$ and $t \in \mathbb{R}$. Fix $x \in \mathcal{M}$ and $t \in \mathbb{R}$. Consider a sphere $\tilde{S}^{s u}(x, r)$ in $\tilde{W}^{s u}(x)$ of a small radius $r$. We estimate the distortion of $\tilde{S}^{s u}(x, r)$ under the action of $\tilde{\varphi}^{t}$. We can choose $r_{0}>0$ such that for all $r, 0<r<r_{0}$,

(i) The image of $\tilde{S}^{s u}(x, r)$ as we pass from $\tilde{W}^{s u}(x)$ to $W^{s u}(x)$ along the flow direction lies between two concentric spheres of radii $k_{1} r<k_{2} r$, where $k_{1}$ and $k_{2}$ are constants depending only on the maximal angle $E^{s u}$ and $\tilde{E}^{s u}$ can form.

(ii) The distortion of $S^{s u}\left(x, k_{2} r\right)$ under the flow $\varphi^{t}$ from $W^{s u}(x)$ to $W^{s u}\left(\tilde{\varphi}^{t} x\right)$ is at most $2 K$, where $S^{s u}\left(x, k_{2} r\right)$ is the sphere of radius $k_{2} r$ in $W^{s u}(x)$ centered at $x$, and $K$ is an upper bound for the quasiconformal distortion of $\varphi^{t}$.

(iii) The image of $\left.S^{s u}\left(\tilde{\varphi}^{t} x, \rho\right)\right)$ as we pass from $W^{s u}\left(\tilde{\varphi}^{t} x\right)$ to $\tilde{W}^{s u}\left(\tilde{\varphi}^{t} x\right)$ along the flow direction lies between two concentric spheres of radii $k_{1} \rho<k_{2} \rho$, for all $\rho, 0<\rho<k_{3}$, where $k_{3}$ is the diameter of the image of $S^{s u}\left(x, k_{2} r_{0}\right)$ under the flow $\varphi^{t}$ from $W^{s u}(x)$ to $W^{s u}\left(\tilde{\varphi}^{t} x\right)$.

Then it is easy to see that the distortion of $\tilde{S}^{s u}(x, r)$ under the action of $\tilde{\varphi}^{t}$ is at most $2 K\left(k_{2} / k_{1}\right)^{2}$ for all $r, 0<r<r_{0}$. Therefore $\tilde{K}(t, x) \leq 2 K\left(k_{2} / k_{1}\right)^{2}$.

We note that while any smooth time change of a conformal Anosov flow is uniformly quasiconformal, it is not necessarily conformal.

\section{Non-stationary Linearization}

In this section we prove a statement on non-stationary linearization. We used a corollary of this statement in the proofs of Theorems 1.3 and 1.4.

Our result is similar to the special case of $1 / 2$ pinching in the theorem on non-stationary normal forms established by A. Katok and M. Guysinsky in [5] and [4]. However, we obtain the statement under a weaker assumption which can be interpreted as a relative or pointwise $1 / 2$ pinching. In particular, this allows us to apply it to uniformly quasiconformal systems. Our statement can also be viewed as a generalization of a one-dimensional result established by A. Katok and J. Lewis in [11]. Our proof closely follows the corresponding steps of the proof in [11].

Proposition 4.1. Let $f$ be a diffeomorphism of a compact Riemannian manifold $\mathcal{M}$, and let $W$ be a continuous invariant foliation with $C^{\infty}$ leaves. Suppose 
that $\left\|\left.d f\right|_{T W}\right\|<1$, and there exist $C>0$ and $\epsilon>0$ such that for any $x \in \mathcal{M}$ and $n \in \mathbb{N}$,

$$
\left\|\left(\left.d f^{n}\right|_{T_{x} W}\right)^{-1}\right\| \cdot\left\|\left.d f^{n}\right|_{T_{x} W}\right\|^{2} \leq C(1-\epsilon)^{n} .
$$

Then for any $x \in \mathcal{M}$ there exists a $C^{\infty}$ diffeomorphism $h_{x}: W(x) \rightarrow T_{x} W$ such that

(i) $h_{f x} \circ f=d f_{x} \circ h_{x}$

(ii) $h_{x}(x)=0$ and $\left(d h_{x}\right)_{x}$ is the identity map,

(iii) $h_{x}$ depends continuously on $x$ in $C^{\infty}$ topology.

Remark 4.1. In the statement, $C(1-\epsilon)^{n}$ can be replaced by $a_{n}$ such that $\sum_{i=1}^{\infty} a_{n}$ converges. The proof works for this case without modifications.

Proof. First we establish uniqueness.

Lemma 4.1. The family $h$ of maps $h_{x}$ satisfying (i), (ii), and (iii) is unique.

Proof. Suppose $\tilde{h}$ is another family of maps satisfying (i)-(iii). Then the family of maps $l=h \circ \tilde{h}^{-1}$ satisfies $l_{f(x)} \circ d f_{x}=d f_{x} \circ l_{x}$, and hence

$$
l_{x}=\left(d f_{x}\right)^{-1} \circ l_{f x} \circ d f_{x}=\cdots=\left(d f_{x}^{n}\right)^{-1} \circ l_{f^{n} x} \circ d f_{x}^{n} .
$$

By the conditions (ii) and (iii), for any $v \in T_{x} W, l_{x}(v)=v+r_{x}(v)$, where $\left\|r_{x}(v)\right\| \leq K\|v\|^{2}$. Therefore,

$$
l_{x}(v)=\left(d f_{x}^{n}\right)^{-1}\left(d f_{x}^{n}(v)+r_{f^{n} x}\left(d f_{x}^{n}(v)\right)\right)=v+\left(d f_{x}^{n}\right)^{-1}\left(r_{f^{n} x}\left(d f_{x}^{n}(v)\right)\right),
$$

and $\left\|\left(d f_{x}^{n}\right)^{-1}\left(r_{f^{n} x}\left(d f_{x}^{n}(v)\right)\right)\right\| \leq\left\|\left(d f_{x}^{n}\right)^{-1}\right\| \cdot K\left\|d f_{x}^{n}(v)\right\|^{2} \leq K C(1-\epsilon)^{n}$. Thus, $\left\|l_{x}(v)-v\right\| \leq K C(1-\epsilon)^{n}$ for any $n \in \mathbb{N}$. This implies that $l_{x}(v)=v$ for any $v \in T_{x} W$ and hence $h=\tilde{h}$.

Our next goal is to obtain a formal power series for $h_{x}$.

Let us fix a small tubular neighborhood $V$ of the zero section in $T W$ such that for each $x$ in $\mathcal{M}$, we can identify the open set $V_{x}=V \cap T_{x} W$ in $T_{x} W$ with a neighborhood of $x$ in the leaf $W(x)$ using the exponential map. The size of this neighborhood can be chosen the same for all $x \in \mathcal{M}$.

Thus we can view the diffeomorphism $f$ as a family of maps $\left.f\right|_{V_{x}}$ from $V_{x} \subset$ $T_{x} W$ to $V_{f x} \subset T_{f x} W$. For each $x \in \mathcal{M}$ we write the (formal) Taylor series of $\left.f\right|_{V_{x}}$ at $0 \in T_{x} W:$ for $v \in V_{x}$

$$
\left.f\right|_{V_{x}}(v)=\sum_{n=1}^{\infty} P_{x}^{n}(v),
$$

where $P_{x}^{n}\left(v_{1}, \ldots, v_{n}\right)$ is an $n$-linear form on $T_{x} W$ with values in $T_{f x} W$, and $P_{x}^{n}(v)$ stands for $P_{x}^{n}(v, \ldots, v)$. Note that $P_{x}^{1}(v)=d f_{x}(v)$. Similarly, we write $h_{x}(v)$ as a formal series

$$
h_{x}(v)=\sum_{n=1}^{\infty} Q_{x}^{n}(v) .
$$

To satisfy the condition (ii), we set $Q^{1}$ to be the identity map. 
We solve for the functions $Q^{n}$ inductively using the commutation relation

$$
h_{f x}\left(f_{x}(v)\right)=d f_{x}\left(h_{x}(v)\right) .
$$

Suppose that we have obtained continuous functions for $Q^{k}, 1 \leq k \leq n-1$. Comparing the homogeneous terms of degree $n$ in the equality

$$
\sum_{n=1}^{\infty} Q_{f x}^{n}\left(\sum_{n=1}^{\infty} P_{x}^{n}(v)\right)=d f_{x}\left(\sum_{n=1}^{\infty} Q_{x}^{n}(v)\right),
$$

we obtain

$$
Q_{f x}^{n}\left(P_{x}^{1}(v)\right)+R_{x}(v)=d f_{x}\left(Q_{x}^{n}(v)\right),
$$

where $R_{x}$ involves only $Q^{i}, 1<i<n$, and $P^{i}, 1<i \leq n$, and thus it is a continuous function on $\mathcal{M}$. Hence

$$
\begin{aligned}
Q_{x}^{n}(v)= & d f_{x}^{-1}\left(R_{x}(v)\right)+d f_{x}^{-1} \circ Q_{f x}^{n}\left(P_{x}^{1}(v)\right)=d f_{x}^{-1}\left(R_{x}(v)\right)+ \\
& \quad+d f_{x}^{-1}\left[d f_{f x}^{-1}\left(R_{f x}\left(P_{x}^{1}(v)\right)\right)+d f_{f x}^{-1} \circ Q_{f^{2} x}^{n}\left(P_{f x}^{1}\left(P_{x}^{1}(v)\right)\right)\right] \\
= & d f_{x}^{-1}\left(R_{x}(v)\right)+\left(d f_{x}^{2}\right)^{-1} \circ R_{f x}\left(d f_{x}(v)\right)+\left(d f_{x}^{2}\right)^{-1} \circ Q_{f^{2} x}^{n}\left(d f_{x}^{2}(v)\right) \\
= & \cdots=\sum_{i=1}^{\infty}\left(d f_{x}^{i}\right)^{-1} \circ R_{f^{i-1} x}\left(d f_{x}^{i-1}(v)\right)
\end{aligned}
$$

To show that the series converges uniformly on $\mathcal{M}$, we estimate the norm of each term. Recall that the norm of a $k$-linear map $P$ is

$$
\|P\|=\sup \left\{\left\|P\left(v_{1}, \ldots, v_{k}\right)\right\|:\left\|v_{j}\right\|=1,1 \leq j \leq k\right\},
$$

and $\left\|P\left(P_{1}, \ldots P_{k}\right)\right\| \leq\|P\| \cdot\left\|P_{1}\right\| \cdots\left\|P_{k}\right\|$. Thus,

$$
\begin{aligned}
\|\left(d f_{x}^{i}\right)^{-1} & \circ R_{f^{i-1} x} \circ d f_{x}^{i-1} \| \\
& \leq\left\|\left(d f_{x}^{i-1}\right)^{-1}\right\| \cdot\left\|d f_{f^{i} x}^{-1}\right\| \cdot\left\|R_{f^{i-1} x}\right\| \cdot\left\|d f_{x}^{i-1}\right\|^{n} \leq K C(1-\epsilon)^{i-1},
\end{aligned}
$$

since $\left\|d f_{f^{i} x}^{-1}\right\|$ and $\left\|R_{f^{i-1} x}\right\|$ are uniformly bounded on $\mathcal{M}$, and

$$
\left\|\left(d f_{x}^{i-1}\right)^{-1}\right\| \cdot\left\|d f_{x}^{i-1}\right\|^{n} \leq C(1-\epsilon)^{i-1}
$$

by (4.1). So the series converges uniformly, and hence $Q^{n}$ is a continuous function on $\mathcal{M}$.

For the rest of the proof, we only need to assume that $\left.f\right|_{W}$ is a contraction.

By a parametric version of Proposition 6.6.3 in [9] there exists a family of smooth functions $\tilde{h}_{x}$ in a neighborhood of the zero section with the above Taylor approximation at 0 , which depend continuously on $x$ in $C^{\infty}$ topology.

The next step is to obtain a family $h$ of $C^{k}$-functions $h_{x}$ which satisfy, in a neighborhood of the zero section, the conditions (i), (ii), and

(iii') $h_{x}$ depends continuously on $x$ in $C^{k}$-topology.

Recall that we fixed a small tubular neighborhood $V$ of the zero section in $T W$ and for each $x$ in $\mathcal{M}$ we identified the open set $V_{x}=V \cap T_{x} W$ in $T_{x} W$ with 
a neighborhood of $x$ in the leaf $W(x)$ using the exponential map. Since $V$ has compact closure we can define

$$
S=\max \left\{\left\|d f_{y}\right\|: y \in V\right\}, \quad s=\max \left\{\left\|d f_{y}^{-1}\right\|: y \in V\right\},
$$

which satisfy $0<s^{-1} \leq S<1$. Here $d f_{y}$ for $y \in V_{x}$ denotes the derivative at point $y$ of the map $\left.f\right|_{V_{x}}$ from $V_{x} \subset T_{x} W$ to $V_{f x} \subset T_{f x} W$ obtained by the above identification.

Let $k$ be an integer such that $k>\log s^{-1} / \log S$. We denote by $B_{V}^{k}$ the space whose elements are families $R$ of $C^{k}$ functions $R_{x}: V_{x} \rightarrow T_{x} W$ such that $R_{x}$ depends continuously on $x$ in $C^{k}$ topology. Let also $A_{V}^{k} \subset B_{V}^{k}$ be the subspace of families $R$ for which $R_{x}$ vanishes to order $k$ at every $x \in \mathcal{N}$, i.e. all terms $R_{x, 0}^{1}, \ldots, R_{x, 0}^{k}$ in the Taylor expansion of $R_{x}$ at $0 \in T_{x} W$ vanish.

For any $x \in \mathcal{M}$ and $y \in V_{x}$ we define $R_{x, y}^{i}$ to be the $i^{\text {th }}$ term in the Taylor expansion of $R_{x}$ at $y$. For $R \in B_{V}^{k}$ and $0 \leq i \leq k$ we set

$$
\|R\|_{V}^{i}=\sup \left\{\left\|R_{x, y}^{i}\right\|: x \in \mathcal{M}, y \in V_{x}\right\} .
$$

Since the derivatives of orders $0 \leq i \leq k-1$ can be inductively estimated in terms of the $k^{t h}$ derivative and the size of the neighborhood, for any $\delta>0$ there exists a neighborhood $V^{\delta} \subset V$ of the zero section such that

$$
\|R\|_{V^{\delta}}^{0}+\|R\|_{V^{\delta}}^{1}+\|R\|_{V^{\delta}}^{k-1} \leq \delta\|R\|_{V^{\delta}}^{k}
$$

for every $R \in A_{V}^{k}$.

We define the operator $\Phi: B_{V}^{k} \rightarrow B_{V}^{k}$ as follows: for any $x$ in $\mathcal{M}$ and $y \in V_{x}$,

$$
(\Phi R)_{x}(y)=\left(d f_{x}\right)^{-1} \circ R_{f x} \circ f(y) .
$$

Lemma 4.2. There exists $\delta>0$ such that the map $\Phi$ is a contraction on the neighborhood $V^{\delta} \subset V$ of the zero section, i.e. for some $\lambda<1$,

$$
\|\Phi R\|_{V^{\delta}}^{k} \leq \lambda\|R\|_{V^{\delta}}^{k} \quad \text { for every } R \in A_{V^{\delta}}^{k} .
$$

Proof. To estimate $\|\Phi R\|_{V^{\delta}}^{k}$, we first calculate the term of order $k, L_{y}^{k}$, in the Taylor approximation of $R_{f x} \circ f: V_{x} \rightarrow V_{f x}$ at $y \in V_{x}$. For $v \in V_{x}$,

$$
R_{f x}(f(v)) \approx \sum_{i=0}^{k} L_{y}^{i}(v) \approx \sum_{i=0}^{k} R_{f x, f y}^{i}(f(v))=\sum_{i=0}^{k} R_{f x, f y}^{i}\left(\sum_{j=0}^{\infty} P_{y}^{j}(v)\right) .
$$

Hence $L_{y}^{k}(v)=R_{f x, f y}^{k}\left(P_{y}^{1}(v)\right)+N_{y}(v)$, where $N_{y}$ is a sum of compositions of $R^{i}$, $i<k$, with $P^{i}, i \leq k$. Since the functions $P^{i}$ are uniformly bounded, it follows from (4.2) that $\left\|N_{y}\right\| \leq M \delta\|R\|_{V^{\delta}}^{k}$ for every $y \in V^{\delta}$. And since $P_{y}^{1}=d f_{y}$, it follows from the definition of $S$ that $\left\|R_{f x, f y}^{k}\left(P_{y}^{1}(v)\right)\right\|_{V^{\delta}} \leq S^{k}\|R\|_{V^{\delta}}^{k}$. Hence, $\left\|L_{y}^{k}\right\| \leq\left(S^{k}\|R\|_{V^{\delta}}^{k}+\delta\|R\|_{V^{\delta}}^{k}\right)$.

The $k^{t h}$ term in the Taylor approximation of $(\Phi R)_{x}$ at $y \in V_{x}$ is $\left(d f_{x}\right)^{-1}\left(L_{y}^{k}\right)$, and hence

$$
\begin{aligned}
\|\Phi R\|_{V^{\delta}}^{k} \leq\left(\sup _{x}\left\|d f_{x}^{-1}\right\|\right) \cdot\left\|L_{y}^{k}\right\| & \leq s\left(S^{k}\|R\|_{V^{\delta}}^{k}+\delta\|R\|_{V^{\delta}}^{k}\right) \\
& =s\left(S^{k}+\delta\right)\|R\|_{V^{\delta}}^{k} .
\end{aligned}
$$


Since $k>\log s^{-1} / \log S$, it follows that $s S^{k}<1$, and we can choose $\delta$ small enough so that

$$
\lambda=s\left(S^{k}+M \delta\right)<1 .
$$

Recall that $\tilde{h}$ is a family of functions $\tilde{h}_{x}$ with the desired derivatives at the zero section. It easily follows from the definition of $\Phi$ that $\Phi \tilde{h}-\tilde{h} \in A_{V}^{k}$. Since $\Phi$ is a contraction on $A_{V^{\delta}}^{k}$, the sequence

$$
h_{n}=\Phi^{n} \tilde{h}=\sum_{i=0}^{n-1} \Phi^{i}(\Phi \tilde{h}-\tilde{h})+\tilde{h}
$$

converges uniformly on $V^{\delta}$ with respect to the norm $\|\cdot\|_{V^{\delta}}^{k}$ to some family $h \in B_{V^{\delta}}^{k}$. Hence $h$ satisfies (iii'). As $\Phi(h)=h$, it follows that $h$ satisfies (i). Since $\tilde{h}$ satisfies (ii), and $h-\tilde{h} \in A_{V^{\delta}}^{k}$, in particular, the first derivative of $h-\tilde{h}$ vanishes at the zero section, we conclude that $h$ also satisfies (ii).

Since $\left.f\right|_{W}$ is a contraction, we can extend $h$ from $V^{\delta}$ to $W$ as follows. For $y \in W_{x}$ we take $n$ such that $f^{n}(y) \in V_{f^{n} x}^{\delta}$ and set

$$
h_{x}(y)=\left(d f_{x}\right)^{-n} \circ h_{f x} \circ f^{n}(y) .
$$

Clearly, $h$ satisfies (i), (ii), and (iii'). Since we can obtain such a solution $h$ for any $k>\log s^{-1} / \log S$, and $h$ is unique, $h_{x}$ is $C^{\infty}$ for every $x \in \mathcal{M}$. This completes the proof of Proposition 4.1.

\section{References}

[1] G. Besson, G. Courtois, S. Gallot, Entropies et rigidités des espaces localement symétriques de courbure strictement négative, GAFA 5 (1995), 731-800.

[2] Y. Benoist, P. Foulon, F. Labourie, Flots d'Anosov à distributions stable et instable différentiables, J. Amer. Math. Soc., 5 (1992), 33-74.

[3] Y. Benoist, F. Labourie, Sur les difféomophismes d'Anosov affines à feuilletages stable et instablediffrentiables, Invent. Math. 11 (1993), 285-308.

[4] M. Guysinsky, The theory of non-stationary normal forms, Ergod. Theory and Dyn. Syst., 22 (2002), 845-862.

[5] M. Guysinsky, A. Katok, Normal forms and invariant geometric structures for dynamical systems with invariant contracting foliations, Math. Research Letters 5 (1998), 149-163.

[6] M. Hirsch, C. Pugh, Smoothness of horocycle foliations, J. Diff. Geom. 10 (1975), 225238.

[7] J.-L. Journé, A regularity lemma for functions of several variables, Revista Mate-mática Iberoamericana 4 (1988), 187-193.

[8] M. Kanai, Differential-geometric studies on dynamics of geodesic and frame flows, Japan. J. Math. 19 (1993), 1-30.

[9] A. Katok, B. Hasselblatt, Introduction to the modern theory of dynamical systems, Encyclopedia of Mathematics and its Applications, 54, Cambridge University Press, LondonNew York, 1995.

[10] R. de la Llave, Rigidity of higher dimensional conformal Anosov systems, Ergod. Theory and Dyn. Syst., 22 (2002), 1845-1870.

[11] A. Katok, J. Lewis, Local rigidity for certain groups of toral automorphisms, Israel J. Math. 75, 203-241. 
[12] C. Pugh, M. Shub, A. Wilkinson, Hölder foliations, Duke Mathematical Journal, 86 (1997), 517-546.

[13] D. Sullivan, On the ergodic theory at infinity of an arbitrary discrete group of hyperbolic motions, in "Riemann surfaces and related topics", Annals of Math. Studies, 97 (1981), 465-497.

[14] P. Tukia, On quasiconformal groups, Jour. d'Analyse Mathe. 46 (1986), 318-346.

[15] C. Yue, Qasiconformality in the geodesic flow of negatively curved manifolds, Geometric and Functional Analysis 6 (1996), 740-750.

Department of Mathematics and Statistics, ILB 325, University of South Alabama, Mobile, AL 36688, USA

E-mail address: sadovska@jaguar1.usouthal.edu 